\title{
On Improvement of Detection of Obstructive Sleep Apnea by Partial Least Square-based Extraction of Dynamic Features
}

\author{
L.M. Sepúlveda-Cano, C.M. Travieso-González, J.I. Godino-Llorente and G. Castellanos-Domínguez
}

\begin{abstract}
This paper presents a methodology for $\mathrm{Ob}$ structive Sleep Apnea (OSA) detection based on the HRV analysis, where as a measure of relevance PLS is used. Besides, two different combining approaches for the selection of the best set of contours are studied. Attained results can be oriented in research focused on finding alternative methods minimizing the HRVderived parameters used for OSA diagnosing, with a diagnostic accuracy comparable to a polysomnogram. For two classes (normal, apnea) the results for PLS are: specificity $90 \%$, sensibility $91 \%$ and accuracy $93.56 \%$.
\end{abstract}

\section{INTRODUGTION}

The obstructive sleep apnea syndrome (OSA) is a common sleep disorder, characterized by obstruction in the airflow. To perform a OSA diagnosis, detection of repetitive episodes of apnea and hypopnea during sleep is carried out, mostly, by attended overnight polysomnography in a sleep laboratory. However, regarding to standard polysomnography test the main disadvantage is the high amount of information required to be analyzed [1], [2].

One of the promising directions for a simple, less costly, noninvasive, reliable and ambulatory screening method for OSA detection is provided by an analysis based on the heart rate variability (HRV) [3]. Nonetheless, analysis framed on the HRV-derived features must deal with nonstationary signals (typical of apnea episodes), making clear the importance of using time-variant or timefrequency representation (TFR) [4]. Almost all extracted features from enhanced TFR, frequently reported, use to be analyzed by static statistical approach, causing the missing of valuable information in the time-evolving process. Conversely, extracted data might be analyzed as stochastically dependent, and thus, there is a need of a feature extraction approach being capable of capturing the dynamic information. In [5], [6], a methodology of feature extraction is discussed doing reference to the analysis of dynamic relevance based on time-adapted linear component approach, which lies on the hypothesis

\footnotetext{
L.M. Sepúlveda-Cano and G. Castellanos-Domínguez are with Control and Digital Signal Processing Group, Universidad Nacional de Colombia, sede Manizales. lmsepulvedac, cgcastellanosdounal . edu.co

C.M. Travieso-González is with University of Las Palmas de Gran Canaria, Technological Centre for Innovation in Communications (CeTIC), Signals and Communications Department, Campus Universitario de Tafira, s/n, Las Palmas de Gran Canaria, Spain. ctraviesoldsc.ulpgo.es

J.I. Godino-Llorente is with Departamento de Ingenieria de Circuitos y Sistemas, Universidad Politénica de Madrid, Spain;. igodinolies. upm.es
}

that each time-dependent characteristic holds a relative associated weight of relevance.

This paper presents a methodology for diagnosing of obstructive sleep apnea from HRV recordings, based on relevance analysis of dynamic features extracted from $t-$ $f$ representation. As a measure of relevance, the Partial Least Squares (PLS) method is used as a supervised multivariate transformation that yields components maximally related with labels [7].

The rest of this paper is organized as follows: first, the time-varying spectral analysis is introduced, then, the methodology for dynamic relevance of dynamic features is described in detail. Lastly, the effectiveness of a feature set based on nonparametric TFR (that represents the dynamics of the HRV activity) is illustrated for the OSA detection through cross-validation using a $k-n n$ classifier, followed by a discussion of the results.

\section{MAterials AND METHODS}

\section{A. Time-frequency representations}

The time-frequency representations are based on the uncertainty principle where the signal frequency in a particular time cannot be determined. Sometimes, both parameters are needed (time and frequency), especially in non stationary signals with dynamic changes. In this way, the Short Time version of the Fourier Transform (termed STFT), introduces a time localization concept by using a tapering window function $\phi$. The spectrogram is a common TFR used in different applications, and it is calculated as the magnitude square of the STFT, as follows:

$$
|\langle x, \phi\rangle|^{2}=\left|\int_{T} x(\tau) \phi(\tau-t) e^{-j 2 \pi f \tau} d \tau\right|^{2} \triangleq \boldsymbol{S}_{x}(t, f),
$$

Another worthy approach to analyze non-stationary signals is to expand them into basis functions, assembled from shifted and scaled versions of a given mother function, but keeping the energy concentrated in short intervals of the $t-f$ plane (referred as wavelets). Nevertheless, the tuning of this type of representation is more complex than the spectrogram and this topic is outside of the scope of the paper.

\section{B. Dynamic features}

A dynamic feature refers to numeric values that represent measures changing over some associated dimension, 
with the purpose of combine the frequency and magnitude information from the power spectrum. In this work, the dynamic features chosen, according with [8] are:

- Linear Frequency Cepstral Coefficients (LFCC), extracted by Discrete Cosine Transform of triangular log-filter banks, $\left\{H_{m}[k]: m=1, \ldots, M\right\}$, linearly spaced in the frequency domain:

$$
c_{r}[n]=\sum_{m=1}^{M} \log \left(s_{m}[n]\right) \cos \left[r\left(m-\frac{1}{2}\right) \frac{\pi}{R}\right]
$$

where $R$ is the number of desired LFCC contours to be considered, and $s_{m}$ is the weighted sum of each filter response set, $s_{m}[n]=\sum_{k=1}^{K} S_{x}[n, k] H_{m}[k]$, being $m, n$ and $k$ indexes for filter ordinal, time and frequency axes, respectively; $K$ stands for the number of samples in the frequency domain.

- Spectral Centroids, that are computed for each filter in the frequency domain, $H_{m}^{\prime}[k]$, by [9] :

$$
\kappa_{m}[n]=\frac{\sum_{k=1}^{K} k H_{m}^{\prime}[k] S_{x}^{\gamma}[n, k]}{\sum_{k=1}^{K} H_{m}^{\prime}[k] S_{x}^{\gamma}[n, k]}
$$

where $\gamma$ is a parameter that represents the dynamic range of the spectrum, used in the computation of the centroid, and the filters $H_{m}^{\prime}[k]$ are linearly distributed along the spectrum.

- Energy of Centroids, is the energy around each centroid. It can be also considered for a fixed bandwidth $\Delta k$ and is computed by means of:

$$
. e_{m}[n]=\sum_{k=\kappa_{m}[n]-\Delta k}^{\kappa_{m}[n]+\Delta k} S_{x}[n, k], \quad 1 \leq m \leq M
$$

\section{Dynamic relevance}

The Partial Least Squares (PLS) regression might be used as a supervised technique, adapted to extract stochastic features from data, building a linear model that describes some predicted variables $\boldsymbol{X}$ in terms of other observable variables $\boldsymbol{Y}$ [7]. The main idea is to find a transformation $\mathscr{G}$, such that: $\boldsymbol{\xi} \mapsto \zeta=\mathscr{G}\{\xi, g\}$, which reduces $p$-dimensional feature vector, $\xi$, to $q-$ dimensional feature vector, $\zeta$, with the best correspondence with its own class. It has the advantage of the extra information given by the label class set $c=\left\{c_{k}\right\}$, when a given relevance function $g\left(\xi_{j} ; c_{k}\right)$ becomes labeldependent. The basis vectors, $\left\{\boldsymbol{\tau}_{i}: i=1, \ldots, q\right\}$ result after simultaneous decomposition:

$$
\begin{cases}\boldsymbol{X} & =\boldsymbol{T} \boldsymbol{P}^{\top}+\varepsilon_{X}, \quad \boldsymbol{T}^{\top} \boldsymbol{T}=\boldsymbol{I} \\ \boldsymbol{Y} & =\boldsymbol{T} \boldsymbol{Q}^{\top}+\varepsilon_{Y}\end{cases}
$$

where matrices $\varepsilon_{X}$ and $\varepsilon_{Y}$ are the error terms, assumed to be i.i.d. normal. $\boldsymbol{P}$ and $\boldsymbol{Q}$ contain the weights used for revealing the influence of individual $\boldsymbol{X}$ and $\boldsymbol{Y}_{-}$ variables, respectively.

\section{EXPERIMENTAL SET-UP}

The proposed methodology for diagnosing obstructive sleep apnea, based on relevance analysis of dynamic features extracted from $t-f$ representation, appraises next stages: First, a preprocessing phase is carried out. In the second phase, the dynamic features are extracted from nonparametric $t-f$ representation based on spectrogram. In the third phase Partial Least Squares (PLS) is used as supervised method to perform dimensionality reduction. In The last phase the results are validated through cross-validation using a $k-n n$ classifier as in [8].

\section{A. Database and Preprocessing}

This collection of electrocardiographic recordings was downloaded from PhysioNet [10], which consists of 30 ECGs, each one including a set of reference annotations obtained from the study of simultaneously recorded respiration signals and added every minute of the recording that indicate either the presence or absence of apnoea during each segment of time. Database is divided into two groups: class Apnea, with at least $100 \mathrm{~min}$ with sleep apnea; and class Normal, with only 5 min or less with sleep apnea (no apnea). These recordings were obtained from men and women between the ages 27 through 63 years old, with weights ranging from 53 to $135 \mathrm{~kg}$. Database holds signals digitized at $100 \mathrm{~Hz}$ with 16 bit resolution. It must be quoted that for comparison sake, training conditions over this database are selected as recommended in [11].

\section{B. TFR Enhancement and Feature Generation}

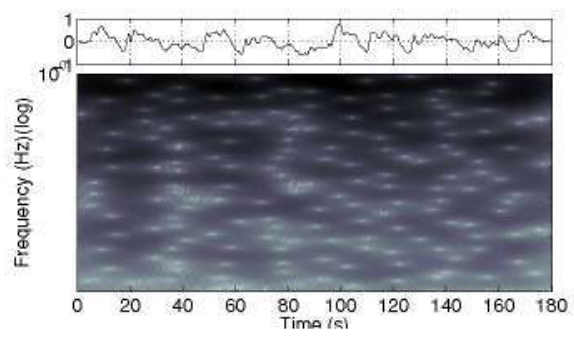

(a) Normal-STFT

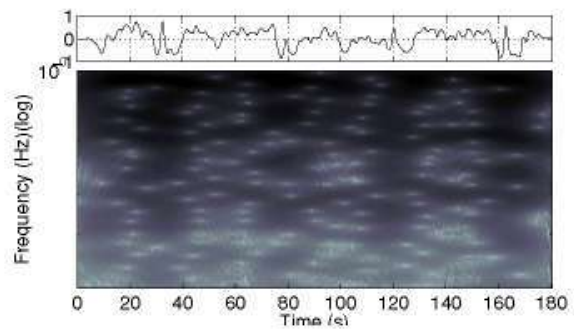

(b) Apnea-STFT

Fig. 1. Examples of TFR on dependence on considered nonparametric enhancement estimated for HRV recordings with labels: normal and apnea.

Figure 1 shows examples of estimated $t-f$ representations, performed for typical normal and pathological 
HRV signals. As usual, the normal case holds the low frequency $(0.04-0.15 \mathrm{~Hz})$ and high frequency $(0.15-$ $0.5 \mathrm{~Hz}$ ) bands of the HRV. Conversely, the pathological representation does not have this high frequency component, but its energy is concentrated around the lower frequencies. For those reasons, the parameters of the representation are a Hamming window of $32.5 \mathrm{~ms}$ length and $50 \%$ of overlapping.

The number of dynamic features chosen is: 20 spectral centroids and their respective energy (4) according with [12], and 12 vectors for cepstral coefficients.

\section{Estimation of relevance weights}

Each dynamic feature is assumed to have a relative associated weight of relevance; the largest weight the most relevant the contour. However, any estimate of relevance weight is conditioned by the given dynamic feature set taken for calculation. For the concrete case of OSA diagnosing, the best set of selected contours can be achieved using, al least, two different combining approaches of comparison: firstly, taking a partially divided set that comprises just a single type of performed dynamic features, having the same principle of generation. Secondly, the best contours are chosen among the whole set of features, no matter on their physical meaning. In this work, both combining approaches of dynamic features are studied in terms of dimension reduction and accuracy performance. Although the former approach is more commonly used because of the convenient physical interpretation of selected set, tuning of training procedures is provided throughout this work based on latter combining way.

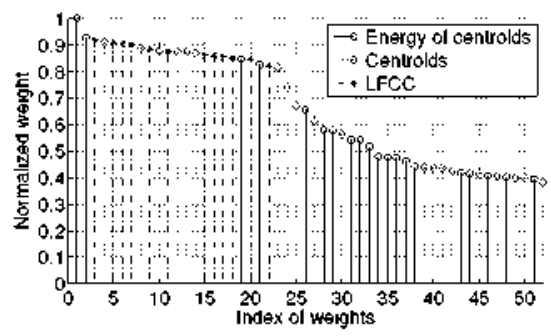

Fig. 2. Computing relevance weights for considered combining approaches of comparison among dynamic features.

From Figure 2, it is possible to infer the advantage of cepstral coefficients contours among another compared dynamic sets because of their higher relevancy weights and the small size of the subset (only 12 features).

\section{Dimension reduction}

Dimension reduction can be achieved selecting the most significative weights (in terms of relevance) instead of adding one by one the features until find the breakpoint of performance. When the amount of features is extensive, a simple classification task leads with a huge computational load. In fact, for the proposed dynamic relevance-based methodology of training, the estimation of the concrete number of selected contours to be considered as the most relevant set remains an open issue [5]. However, it had been established that based of the above fixed changing behavior, the performance breakpoints could be found, assuming the ordinal of the respective weight as a coarse estimation of the needed number of relevance weights.

With this in mind, the normalized difference between two consecutive ordered by relevance weights, as plotted in the Figure 3 , gives better representation of changing behavior, where the local minimum is fixed to be the breakpoint and, therefore, pointing out on the number of selected contours to be considered as the most relevant set. In this case, the number of dynamic features needed is around 34 .

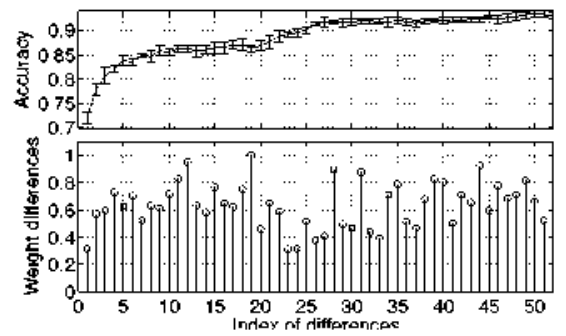

Fig. 3. Performed accuracy dependence on normalized difference between two consecutive ordered by relevance weights for considered TFR enhancement.

Regarding to comparison among partially divided sets of dynamic features, Figure 4 depicts estimated accuracy values of time-dependent contours, which are estimated by adding one by one their weights ordered by decreasingly relevance. It can be seen that the subset of cepstral coefficients achieves better accuracy, according with relevance analysis.

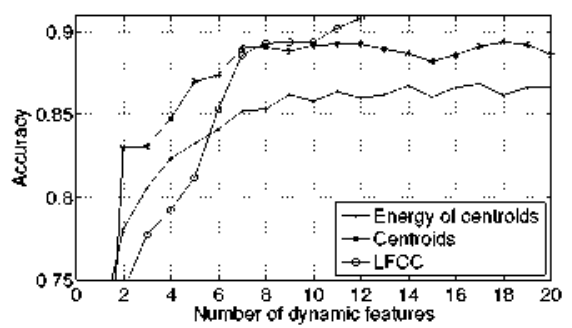

Fig. 4. Accuracy performance for partially divided sets of selected dynamic features

\section{RESULTS}

Table I shows the results for the best configuration for the full set and the partial set and its respective parameters. Figure 5 shows the Receiving Operating Curve (ROC) for the same configuration. The best performance is achieved by the full set for dynamic features, nevertheless, the computational cost is improved by the partial set with an acceptable performance. 
TABLE I

PERFORMANCE OF CLASSIFIER FOR BEST CONFIGURATION

\begin{tabular}{|c||c||c|}
\hline Set & Accuracy & Number of components \\
\hline$L F C C$ & $91 \%$ & 13 \\
\hline All & $93.56 \%$ & 21 \\
\hline
\end{tabular}

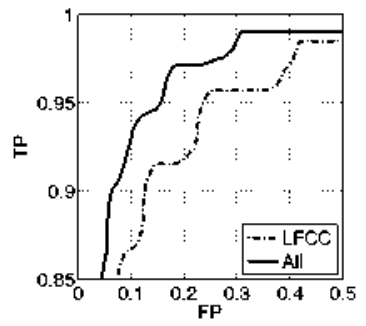

Fig. 5. Receiving Operating Gurve for bests configurations

Table II shows the sensibility and specificity for the same test, both measures indicate that the full set of dynamic features presents the best performance.

TABLE II

SENSIBILITY AND SPECIFICITY FOR BESTS CONFIGURATIONS

\begin{tabular}{|c||c||c||c||c|}
\hline Set & Sensibility & Specificity & Precision & Error-mean \\
\hline$L F C C$ & $92 \%$ & $87 \%$ & $88 \%$ & $10 \%$ \\
\hline All & $91 \%$ & $90 \%$ & $90 \%$ & $9 \%$ \\
\hline
\end{tabular}

\section{CONCLUSIONS AND FUTURE WORK}

The training methodology is explored, which is based on relevance analysis by PLS as an alternative technique for OSA detection. Both considered methods of feature extraction (partial and full set) presents an accuracy performance over $90 \%(93.56 \%$ in the full set case) and a reduction dimension significant. The number of dynamic features needed for classification task is around 34 (18 less than original set) and the number of components required after the linear transformation is about 21 for all features and 13 for LFCC subset (9 and 17 less than original number of components respectively). In [8] is reported an accuracy of $92.67 \%$ using SPWVD as TFR and relevance analysis by PCA; in this way, the advantage of the supervised method proposed in this paper to get a better estimation of relevance measure and a suitable transformation is evident. As future work, further efforts finding an alternative for OSA diagnosing, having the added benefit of low cost and simplicity, should be focused on extended studies over different ECG databases and another approaches in conjunction with HRV analysis [11] (by instance, photopletysmography signal [13] or ECG-derived respiration signal [14]).

\section{ACKNOWLEDGMENTS}

This research is partially supported within the framework of "Servicio de monitoreo remoto de actividad car- diaca para el tamizaje clínico en la red de telemedicina del Departamento de Caldas" financed by "Proyectos de investigación e innovación conjuntos entre grupos de trabajo académico, Universidad de Caldas y Universidad Nacional Sede Manizales, hacia tercer milenio"; and by private funds from Spanish Company, Telefónica, under the project called "Cátedra Teléfonica-ULPGC 2009" grant.

\section{REFERENCES}

[1] E. Sheridan R. Reilly P. Nolan P. Chazal, C. Heneghan and M. O'Malley. Automated processing of the single-lead electrocardiogram for the detection of obstructive sleep apnoea. IEEE Thansactions on Biomedical Engineering, 5046):686-696, 2003.

[2] I. Cosic D. Cvetkovic H. Abdullah, G. Holland. Correlation of sleep eeg frequency bands and heart rate variability. In 31st Annual International Conference of the IEEE EMBS, pages 5014-5017, 2009 .

[3] Zhi-De Deng, Chi-Sang Poon, N.M. Arzeno, and E.S. Katz. Heart rate variability in pediatric obstructive sleep apnea. In Engi. neering in Medicine and Biology Society, 20(16. EMBS '06. 28th Annial International Conference of the IEEE, pages $3565-3568$, aug. 2006.

[4] SV. Huffel M. Matteucci T. Penzel S. Cerutti M. Mendez, $\mathrm{J}$ Corthout and AM. Bianchi. Automatic screening of obstructive sleep apnea from the ecg based on empirical mode decomposition and wavelet analysis. Physiol. Meas, 31:273-289, 2010.

[5] A. Quiceno-Manrique, I. Godino-Llorente, M. Blanco-Velasco, and G. Castellanos-Dominguez. Selection of dynamic features based on time-frequency representations for heart murmur detection from phonocardiographic signals. Ann Biomed Eng., 38, 200\%.

[6] M. Blanco-Velaseo G. Castellanos-Dominguez LD. AvendañoValencia, JI. Godino-Llorente. Feature extraction from parametric time frequency representations for heart murmur detection. An. nals of Biomedical Engineering, accepted for publicaction, 2010.

[7] M. Barker and W. Rayens. Partial least squares for discrimination. Journal of chemometrics, $17(3): 166-173,2003$.

[8] C.M. Travieso-Gonzalez M.A. Ferrer-Ballester A.F. QuicenoManrique, J.B. Alonso-Hernandez and G. Castellanos-Dominguez. Detection of obstructive sleep apnea in ecg recordings using timefrequency distributions and dynamic features. In 31st Annual International Conference of the IEEE EMBS, 2009.

[9] B. Gajic and K. Paliwal. Robust feature extraction using subband spectral centroid histograms. In in Proc. ICASSP, pages 85-88, 2001.

[10] T. Penzel. Is heart rate variability the simple solution to diagnose sleep apnoea? Eur Respir $J, 22(6): 870-871$, Dec 2003.

[11] L. Rodríguez-Linares AJ. Méndez DN. Olivieri MJ. Lado, XA. Vila and P. Félix. Detecting sleep apnea by heart rate variability analysis: Assessing the validity of databases and algorithms. Journal. of Medical Systems, DOI:10.1007/s10916-009-9383-5, 2009.

[12] K. Howorka, J. Pumprla, A. Jirkovskac, S. Lacigovad, and J. Nolan. Modified orthostatic load for spectral analysis of shortterm heart rate variability improves the sensitivity of autonomic dysfunction assessment. Journal of Diabetes and Its Complications, 24:48-54, 2010.

[13] JM. Vergara-S. Cerutti AM. Bianchi E. Gil, M. Mendez and P. Laguna. Discrimination of sleep-apnea-related decreases in the amplitude fluctuations of ppg signal in children by hrv analysis. IEEE Thansactions On Biomedical Engineering, 56(4):1005-1014, 2009.

[14] M. Palaniswami AH. Khandoker and CK. Karmakar. Support. vector machines for automated recognition of obstructive sleep apnea syndrome from ecg recordings. IEEE Trans Inf Technol Biomed, 13(1):37-48, Jan 2009. 